\title{
Interoception: An Overview for Allied Health Professionals
}

\author{
Geela Spira* \\ Department of Occupational Science and Occupational Therapy, Eastern Kentucky University, USA
}

*Corresponding author: Geela Spira, PhD, OTR/L, Department of Occupational Science and Occupational Therapy, Eastern Kentucky University, USA. E-mail: Geela.spira@eku.edu

Received Date: October 21, 2019

Published Date: October 31, 2019

\begin{abstract}
The concept of Interoception and Interoceptive Awareness is being investigated and used clinically by a variety of scientists and health care professionals. Few peer- reviewed studies are found in the literature by allied health care professionals. It may be useful for allied health care professionals to access a paper summarizing and explaining the concept in terms that are aimed for their use. The paper reviews basic science concepts of interoception, interoceptive awareness, models to explain the dynamics of aspects of interoception, and the application to intervention approaches in the allied health professions.
\end{abstract}

Keywords: Interoception; Emotional regulation; Metacognition; Occupational therapy; Allied health

\section{Introduction}

Interoception is a newly emerging field which spans multiple disciplines from basic science to holistic interventions. As the subject is investigated, further elaborations and ways to view the dynamics are emerging from the literature. From foundational science to health and well-being, interoception spans concepts from homeostasis to insight and adaptive behavior. This paper aims to provide an introductory synopsis of the topic that is geared towards allied health professionals.

Although the term 'interoception' has been in use for over one hundred years, the term then referred to sensory receptors that provided internal sensory feedback to the body, and not the sensory receptors that provided external sensory feedback to the body (exteroception) [1]. In 2002, according to Strigo IA Craig AD et al. [1], a broader concept was proposed by scientists which also included the ascending pathway to the cortex which "conveyed homeostatic sensory activity from all bodily tissues" and interacted with the central autonomic network.

As psychology and health care professionals began to consider interoception, concepts linking sensory feedback to cortical oversight, emotional awareness and regulation, and general feeling states were added to the definition [2]. The addition of conscious thought offered a way to intervene and change one's perceptions of the 'embodied experience' Farb $\mathrm{N}$ et al. [3], and so the concept of interoceptive awareness was added to the lexicon. Interoceptive awareness can be defined as the processing of inner sensations so that they become available to conscious awareness Farb $\mathrm{N}$ et al. [3], Cameron O [4] \& Price CJ \& Hooven C [5] propose that interoceptive ability implies having capacity to "positively manage challenging sensations and related behavioral responses" by moderating, suppressing, or changing signals. The authors further considered that interoceptive awareness will lead a person to interpret and contemplate one's interoceptive experiences. This opened the way for holistic practitioners to work on interoceptive perceptions and biases through holistic contemplative practices, with the goal of "restoring a person's sense of presence and agency in the world" ( $p$. 1). One such example is the psychology-based 'Mindful Awareness in Body- Oriented Therapy' (MABT), which promotes the practice of mindful body awareness [5].

\section{Basic Models of Interoception}

\section{Allostatic load model}

Allostasis is an expanded concept of homeostasis. Not only does the body regulate and maintain constancy within body systems, allostasis adaptively readjusts the regulatory levels in response to changing physiological demands, typically considered 'stressors' [6]. 
The Allostatic load model states that stressors in the environment lead to a dysregulation of the body's Stress Response System (SRS). The SRS is a primary integrative pathway in which "psychosocial environmental factors are transmuted into behavioral, autonomic and immunologic adaptation" or into pathology [5,7]. Although the SRS initially reacts to stressors with hyper-vigilance, over time the system may become less sensitive and responsive in order to manage the chronic stress. While adaptive, this process may decrease interoceptive ability to detect and interpret bodily cues.

\section{Adaptive Calibration model}

Adaptation to stress is desirable in momentary situations. The adaptation may become learned for survival needs and become a set pattern of response. Patterns are typically set during early life events and determine one's autonomic responsivity well after the precipitating events. However, difficulty arises when the environmental challenges change, and the SRS does not adaptively change to the new demands [8]. The dynamic of being prepared and safe by reverting to automatic patterns of responsiveness is referred to as 'buffering' the organism by establishing physiological 'set points' that "buffer signals and protect the organism $[5,8]$. The overall adaptivity of set points is weighed against the need for responding to the actual environmental situation in one's present. The more one relies on set points, the less awareness and interpretation of feelings in the challenging situation occurs. Therapeutic approaches, such as mindfulness or yoga, would focus on reshaping the interoceptive awareness to the occurring environmental cues and one's physiologic response in order to increase regulation of one's state, both physiologically and emotionally $[3,5]$.

\section{Applied Models of Interoception}

\section{Predictive coding model of Interoception}

Demasio A [9] expounded on the concept of a 'simulation map' in which regulation is achieved through a neural representation of the body in a stable representation albeit abstract interpretation of one's sensory experiences. The simulation map is in contrast to the direct experience of one's sensory experiences in the 'here and now'. A simulation map may be thought of as 'a person's sense of embodiment in the world' [3]. The simulation map may explain our pre-disposed motivations to action, as a person resolves current challenges through comparison to the internal predictive abstract concept of one's interoceptive milieu.

The Predictive Coding model of Interoception posits that the simulation map resolves interactions between the body and its external environment, or between the current sensation of the body and the expectations of the body based on past experiences [3]. The simulation map is considered to be largely inaccessible to conscious experience. So, when incoming sensation diverges from the prior existing simulation map, the organism may either update that layer of the simulation map to match with the new sensory condition, or the organism may regulate the new sensation to fit with the prior existing map. The use of higher order cognition in this process will depend on how high, and thus motivating, the discrepancy is between the current sensation and the simulation map's prior existing representation within the body [10].

The Predictive Coding model compares between the sensed and the expected states in order to choose an action. Two possible actions are posited by this model. Active inference will prompt the organism to action, such as taking a medication, in order to shape the immediate interoceptive milieu to match the expected state. This is considered a 'bottom-up' perceptual state of being. The second action is perceptual inference, which is a cognitive strategy in which a 'top-down' strategy will be used to accept the immediate sensation. Perceptual inference does not seek to change the immediate milieu, but rather updates the expectations of simulation map, such as in accepting certain levels of pain $[3,10]$.

The model suggests that the most effective behavioral adaptation would be 'iterative cycling between perceptual and active inferences. The tradeoff is between noticing specifics of one's internal sensory experiences and having an organized and regulated predictable pattern of habitual response. The model proposes that the most efficient balance is to be able to use flexibility in the iterative shifting 'back and forth between sensory monitoring and conceptual inference' [3]. Therapies which promote contemplation would be useful in this model as they can challenge the cognitive dampening of immediate interoception by reducing obligatory conditioned appraisals and responses to current situations, by emphasizing the current perception of one's interoceptive sensations during that phase of the appraisal process.

\section{Behavioral measurement of Interoception}

Measurement of interoception has advanced. Current measures of interoception partition abilities into three categories. Objectives measures of interoceptive performance include behavioral scores on heartbeat detection tasks. Subjective measures of interoceptive perception include self-rated questionnaires of how well one perceives they interpret interoceptive signals.

Critchley HD \& Garfinkel SN [2] posit a third measure of higher order metacognitive interoception, called interoceptive insight, in which actual objective performance is compared to the subjective sense.

\section{Intervention}

Given the delineated models, several possibilities for intervention by allied health professionals are noted. One construct is that of mismatch or accuracy between the perceived sensitivity to one's interoceptive state and one's accuracy noted in the performance of an interoceptive task, such as heartbeat detection, and that of sustained attention. Garfinkel SN, et al. [11] found that a higher mismatch in people with Autism Spectrum Disorder (ASD) predicted symptoms of anxiety and deficits in interpersonal emotional interaction. Schauder KB, et al. [12] noted no significant differences in interoceptive awareness in performance tasks, however the ASD group was found to have a significantly heightened sustained attention to their internal interoceptive state over time. 
The authors suggested that heightened sustained attention to internal cues may have been maladaptive, such as is found in anxiety and depression. Additionally, the heightened attention to internal cues was suggested to be a contributing factor in decreased attention to external environmental stimuli, which also affected decreased social interaction $[12,13]$.

Another construct is mindful awareness. Client education to promote mindful awareness is used in the MABT Explanatory model. The program begins with Awareness of interoception in order to achieve 'body literacy'. The program then strives to improve Access to interoceptive states through training exercises for awareness and reduction of distress. Finally, MABT model educates clients to Appraise their interceptive state by practicing mindful body awareness. This stage aims to improve emotional regulation and promote resilience [5].

Other therapies are mentioned as appropriate for use in mindfulness and awareness of interoception (Farb, 2015). These therapies include therapies that pay attention to specific body sensations or use such as yoga and tai chi. Other noted bodyoriented healing and psychotherapy methods were Feldenkrais Buchanan PA \& Ulrich BD [14], the Alexander method Woodman JP \& Moore NR [15], Sensory Awareness Selver C et al. [16], Dialectical-Behavioral therapy Linehan MM et al. [17], and Somatic Experiencing [18].

Evidence-based practice for interoception is increasing as foundational knowledge in the literature expands. Understanding of the mechanisms of how the sensitivity and accuracy of interoceptive signals influence emotional and motivational cues to action will focus expected and desired outcomes. Interventions can influence adaptivity by promoting increased interoceptive awareness, and through balancing interoceptive awareness with representational internal interoceptive maps for stability of the organism. Bakal D, et al. [19] suggests that awareness of the interoceptive process can be used by physicians and patients to determine which allied health professions might "best strengthen the patient's self-management skills and goal of wellness". Allied health professionals have tools to include intervention that incorporates interoception into their clinical practice and into research studies in order to add to the body of evidence addressing this issue in our professions.

\section{Acknowledgment}

None.

\section{Conflict of Interest}

No conflict of interest.

\section{References}

1. Strigo IA, Craig AD (2016) Interoception, homeostatic emotions and sympathovagal balance. Philos Trans R Soc Lond B Biol Sci 371(1708).

2. Critchley HD, Garfinkel SN (2017) Interoception and emotion. Current Opinion in Psychology, 17: 7-14.

3. Farb N, Daubenmier J, Price CJ, Gard T, Kerr C, et al. (2015) Interoception, contemplative practice, and health. Front Psychol 6: 763.

4. Cameron 0 (2001) Interoception: the inside story - a model for psychosomatic processes. Psychosom Med 63(5): 697-710.

5. Price CJ, Hooven C (2018) Interoceptive awareness skills for emotion regulation: Theory and approach of mindful awareness in body-oriented therapy (MABT). Front Psychol 9: 798.

6. Cacioppo JT, Tassinary LG, Berntson GG (2000) Handbook of psychophysiology. $2^{\text {nd }}(\mathrm{edn})$. Cambridge University Press, UK.

7. Ellis BJ, Boyce W, Belsky J, Bakermans Kranenburg M, van Ijzendoorn M (2011) Differential susceptibility to the environment: an evolutionaryneurodevelopmental theory. Dev Psychopathol 23(1): 7-28.

8. Del Giudice M, Ellis BJ, Shirtcliff, EA (2011) The adaptive alibration model of stress responsivity. Neurosci Biobehav Rev 35(7): 1562-1592.

9. Damasio A (2003) The person within. Nature 423(6937): 227.

10. Seth A K, Suzuki K, Critchley HD (2012) An interoceptive predictive coding model of conscious presence. Front Psychol 2: 395.

11. Garfinkel SN, Tilly C, O Keeffe S, Harrison NA, Seth AK, et al. (2016) Discrepancies between dimensions of interoception in autism: implications for emotion and anxiety. Biol Psychol 114: 117-126.

12. Schauder KB, Mash LE, Bryant LK, Cascio CJ (2015) Interoceptive ability and body awareness in autism spectrum disorder. J Exp Child Psychol 131: $193-200$

13. Baranek GT, Watson LR, Boyd BA, Poe MD, David FJ, et al. (2013) Hyporesponsiveness to social and nonsocial sensory stimuli in children with autism, children with developmental delays, and typically developing children. Dev Psychopathol 25(2): 307-320.

14. Buchanan PA, Ulrich BD (2001) The Feldenkrais Method: a dynamic approach to changing motor behavior. Res Q Exerc Sport 72(4): 315-323.

15. Woodman JP, Moore NR (2012) Evidence for the effectiveness of Alexander Technique lessons in medical and health-related conditions: a systematic review. Int J Clin Pract 66(1): 98-112.

16. Selver C, Brooks CVW, Lowe R, Laeng Gilliatt S (2007) Reclaiming Vitality and Presence: Sensory Awareness as a Practice for Life. Berkeley, CA (edn): North Atlantic Books, USA.

17. Linehan MM, Schmidt H, Dimeff LA, Craft JC, Kanter J, et al. (1999) Dialectical behavior therapy for patients with borderline personality disorder and drug-dependence. Am J Addict 8(4): 279-292.

18. Payne P, Levine PA, Crane Godreau MA (2015) Somatic experiencing: using interoception and proprioception as core elements of trauma therapy. Front Psychol 6: 93.

19. Bakal D, Coll P, Schaefer (2008) Somatic awareness in the clinical care of patients with body distress symptoms. Biopsychosoc Med 2(6): 1-6. 